\title{
Molecular Subtypes of Breast Cancer Patients According to St Gallen Classification
}

\author{
Shafatuijahan ${ }^{1 *}$ \\ Ifatujiahan ${ }^{2}$ \\ Rajat Sanker Roy Biswas ${ }^{3}$
}

IDepartment of Oncology
Chattogram Maa-O-Shishu Hospital Medical College
Chattogram, Bangladesh.
${ }^{2}$ Parkview Hospital Private Limited
Chattogram, Bangladesh.
${ }^{3}$ Department of Medicine
Chattogram Maa-O-Shishu Hospital Medical College
Chattogram, Bangladesh.
*Correspondence to:

\section{Dr Shafatuijahan}

Assistant Professor

Department of Oncology

Chattogram Maa-O-Shishu Hospital Medical College Chattogram, Bangladesh.

Mobile: +8801883570210

01717418885

Email: shafatujiahan27@gmail.com

Date of Submission : 05.09 .2019

Date of Acceptance : $\quad 09.10 .2019$

www.banglajol.info/index.php/CMOSHMCJ

\begin{abstract}
Introduction: Breast cancer is a common malignancy among female in Bangladesh. But its molecular subtypes are not evaluated due to lack of expert investigation support. So objectives of the present study are to evaluate the molecular subtypes of breast cancer patients according to St Gallen classification in our contest.
\end{abstract}

Materials and methods: It is retrospective study done among histopathologically proved 40 breast cancer patients visiting Medical Oncology and Radiotherapy department of Chattogram Maa-O-Shishu Hospital. Molecular subtypes was evaluated by immunohistochemistry according to St Gallen Classification.

Results: In this study a total of 40 cases of invasive female breast cancers were included. Age of the patients ranged from 31-62 years, with a mean age of $41 \pm$ 13.5 years. ER expression was seen in $60 \%$ and $P R$ in $55 \%$ of cases and Her-2/neu positivity in $16 \%$. Majority (52.5\%) of the tumors were located in the left breast. The percentage of ER but not PR positivity increased with age, though this difference was not statistically significant. Majority of the cases were diagnosed at stage II with a percentage of $42.5 \%$. Stage II tumors showed more ER and PR positivity. Among all $57.9 \%$ of ER positive and $49.5 \%$ of $P R$ positive tumors were present while $72.2 \%$ of tumors were negative for Her-2/neu. The triple-negative breast tumors were more commonly found at grade 2. Regarding luminal status 14(35\%) was Luminal A, 5(12.5\%) was Luminal B, 9(22.5\%) was TNBC and 12(30\%) was HER 2 positive.

Conclusion: In this study luminal A was the commonest molecular subtypes. Luminal A subtypes tumors had a long term risk of distant matastatic disease which can be reduced by hormonal treatment.

Key words : Breast cancer; Female; Histopathology.

\section{INTRODUCTION}

Breast cancer is most frequently diagnosed cancer of women in Bangladesh and worldwide and leading cause of cancer mortality ${ }^{1,2}$.

In Bangladesh with 32.8 percent of female cancer patients suffering from this disease $^{3}$. According to a WHO report only 11 percent of Bangladeshi women is diagnosed breast cancer at an early stage, due to poor access to medical facilities, social stigma and a lack of awareness about the disease $\mathrm{e}^{4}$.

Incidence of breast cancer in Bangladesh is 22.5/100000 population and it has got highest prevalence rate of 19.3/100000 among women of 15-44 years. Traditional classification of breast cancer is based on the clinicopathological analysis of tumors, with classification by based on histopathological features, including the pattern of architectural growth (eg, cribriform, papillary) and the nuclear grade (Low, intermediate, or high $)^{5}$. Treatment choices depends on tumor size, local invasion and 
lymph node involvement or distant metastases, as defined by the American Joint Committee on Cancer's TNM staging classification ${ }^{6}$. Histological type, grade, tumor size, lymph node involvement and Estrogen Receptor (ER) Progesterone Receptor (PR) and HER-2 neu receptor status all has prognostic and predictive value.

A new classification system for breast cancer subtypes presented in St. Gallen, breast cancer divides Breast cancer in four main categories in Luminal A, Luminal B with HER2 negative, Luminal B with HER2 positive, HER2 enriched and basal-like (Triple negative) ${ }^{7}$.

In these studies we intended to show the Immunohistochemical pattern of breast cancer patients visiting a tertiary care hospital. Luminal A breast cancer is hormone-receptor positive (Estrogen-receptor and/or progesterone-receptor positive) HER2 negative and has low levels of the protein Ki-67, which helps control how fast cancer cells grow. Luminal A cancers are low-grade, tend to grow slowly and have the best prognosis.

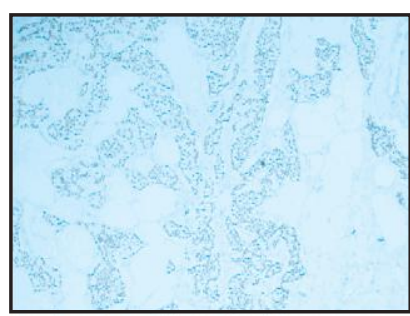

Figure 1 : ER: Nuclear staining in tumor cells for ER

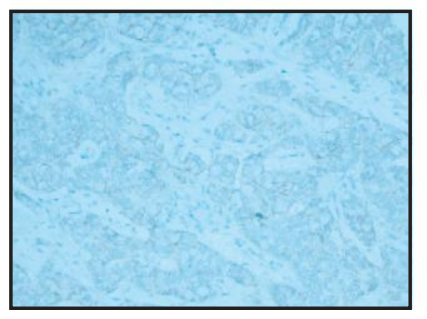

Figure 3 : HER-2/neu: Complete, moderate circumferential membrane staining for HER-2/neu protein

Luminal B breast cancer is hormone-receptor positive (Estrogen-receptor and/or progesterone-receptor positive) and either HER2 positive or HER2 negative with high levels of Ki67. Luminal B cancers generally grow slightly faster than luminal A cancers and their prognosis is slightly worse.

Triple-negative / basal-like breast cancer is hormone-receptor negative (estrogen-receptor and progesterone-receptor negative) and HER 2 negative. This type of cancer is more common in women with BRCA1 gene mutations. Researchers aren't sure why, but this type of cancer also is more common among younger and African-American women.

HER2-enriched breast cancer is hormone-receptor negative (Estrogen-receptor and progesterone-receptor negative) and HER2 positive. HER2-enriched cancers tend to grow faster than luminal cancers and can have a worse prognosis, but they are often successfully treated with targeted therapies aimed at the HER2 protein (Such as trustuzumab, pertuzumab, lapatinib, neratinib and T-DM1 or ado-trastuzumab emtansine).

\section{MATERIALS AND METHODS}

This was a retrospective study done in the Department of Medical, Oncology and Radiotehrapy at Chattogram MaaShishu-O-General Hospital during a study period of three months. A total of 40 histopathologically confirmed breast cancer patients of Chattogram Maa Shishu O General Hospital were included in the study after getting the ERB clearance of the hospital. Cancer registry file was explored to get the necessary information and data was compiled and analyzed by SPSS version 20.

The specimen was received in the Pathology department in $10 \%$ formalin. In every case the standard protocol for surgical grossing of the specimens was followed. After a detailed specimen description, multiple sections were taken after conventional processing, paraffin sections of $5 \mu \mathrm{m}$ thickness were stained by Haematoxylin and Eosin ( $\mathrm{H} \& \mathrm{E})$ for histopathological study. In addition, $3 \mu \mathrm{m}$ sections was cut from a paraffin block of tumor tissue and taken on 4 glass slides coated with adhesive (Silane) for Immunohistochemistry (IHC) to detect $\mathrm{Ki} 67, \mathrm{ER}, \mathrm{PR}$, and HER2/neu expression. The technique for IHC included antigen retrieval in this buffer in a microwave oven, blocking endogenous peroxidase with $3 \%$ hydrogen peroxide, incubating with primary mouse monoclonal antibody ( Novocastra, Leica ), linking with rabbit anti mouse secondary antibody (Novocastra, Leica) enzyme labelling with streptavidin- horseradish peroxidase (Novocastra, Leica), developing chromogen with Deaminobenzidine (DAB) and counterstaining with haematoxylin.

\section{RESULTS}

Table I : Patient profile of cancer

\begin{tabular}{llrr} 
Variable & Type & Frequency & Percentage \\
Site of breast cancer & right & 17 & 42.5 \\
& Left & 21 & 52.5 \\
\multirow{3}{*}{ Lymph node } & bilateral & 2 & 5 \\
\multirow{3}{*}{ Stage } & positive & 21 & 52.5 \\
& negative & 19 & 47.5 \\
& Stage I & 10 & 25.0 \\
Histopathology & Stage II & 17 & 42.5 \\
& Stage III & 7 & 17.5 \\
& Stage IV & 6 & 15.0 \\
& Ductal cell carcinoma & 19 & 47.5 \\
Grade & Medullary carcinoma & 1 & 2.5 \\
& Infiltrating type & 19 & 47.5 \\
& Colloid type & 1 & 2.5 \\
Surgery & 1 & 19 & 47.5 \\
& 2 & 18 & 45.0 \\
& 3 & 3 & 7.5 \\
& Simple mastectomy & 4 & 10.0 \\
& Simple mastectomy with & & \\
& axillary clearance & 21 & 52.5 \\
& Radical mastectomy with & & \\
& axillary clearance & 5 & 12.5 \\
& Lumpectomy & 2 & 5.0
\end{tabular}




\begin{tabular}{llrr} 
& No surgery done & 8 & 20.0 \\
radiotherapy & Given & 15 & 37.5 \\
& Not given & 25 & 62.5 \\
chemotherapy & Given & & \\
& Not given & & \\
ER status & Positive & 24 & 60.0 \\
& Negative & 16 & 40.0 \\
PR status & Positive & 22 & 55.0 \\
& Negative & 18 & 45.0 \\
Her 2 status & Positive & 16 & 40.0 \\
& Negative & 24 & 60.0 \\
Ki 67 & Positive & 12 & 30 \\
& Negative & 19 & 48 \\
Age & Nil & 9 & 22 \\
\hline
\end{tabular}

Table I showing 40 cases of invasive female breast cancers were included in our study. The age of the patients ranged from 31-62 years, with a mean age of $41 \pm 13.5$ years. ER expression was seen in $60 \%$ and PR in $55 \%$ of cases and HER-2/neu positivity in $16 \%$. Majority $(52.5 \%)$ of the tumors were located in the left breast. The clinical features and various combinations of ER and PR receptors are displayed in table I. ER expression correlated well with PR expression. The percentage of ER but not PR positivity increased with age, though this difference was not statistically significant. Majority of the cases were diagnosed at stage II with a percentage of 42.5\%. Stage II tumors showed more ER and PR positivity. However, no significant variation was seen with the HER-2/neu expression. HER-2/neu positive tumors showed a higher mitotic index compared to the negative ones. The reverse trend was seen with mitotic index of ER and PR tumors IDC category, $57.9 \%$ of ER+ and $49.5 \%$ of PR + tumors were present while $72.2 \%$ of tumors were negative for HER-2/neu. The triplenegative breast tumors were more commonly grade 2 . The triple-negative breast cancers characterized by a lack of expression of ER, PR and HER2/neu.

Table II : Molecular subtypes

\begin{tabular}{lcc} 
& Frequency & Percent $(\%)$ \\
Luminal A & 14 & 35.0 \\
Luminal B & 5 & 12.5 \\
TNBC & 9 & 22.5 \\
HER 2 & 12 & 30.0 \\
Total & 40 & 100.0 \\
\hline
\end{tabular}

Regarding luminal status 14(35\%) was Luminal A, 5(12.5\%) was Luminal B, 9(22.5\%) was TNBC and $12(30 \%)$ was HER 2 positive.

\section{DISCUSSION}

Breast cancer is a multifaceted disease having distinct biological subtypes with diverse natural history, presenting a varied spectrum of clinical, pathologic and molecular features with different prognostic and therapeutic implications ${ }^{9}$. Only about one half of patients with early breast cancer are treated and cured by local surgical excision alone. Therefore, it is important to identify the set of patients in whom the disease is destined to recur and which patients are likely to benefit from systemic chemotherapy ${ }^{10,11}$.

In our study we found the age of the patients ranged from 3162 years, with a mean age of $41 \pm 13.5$ years. In a study conducted by Onitilo et al which included 1134 cases of invasive breast carcinomas, it was observed that cases with ER, PR positive and HER2 neu negative subtype were more likely to be older, have early stage breast carcinoma, present with smaller tumor and have a well/moderately differentiated histological grade ${ }^{12}$. They were less likely be lymph node positive, have a lobular phenotype and be treated with chemotherapy. Studies by Ruibal et al and Thike et al showed an association of the histological tumor grade with the ER/PR content $^{11,13}$. A transition from lower to higher histological grades was accomplished by a decrease in ER/PR content. In a study conducted by Rao et al the prevalence of ER, PR and HER2/neu over expression among Indian patients did not fall within the ranges given in literature ${ }^{14}$. The tumor size and grade was related to expression of only ER. Their results showed very high proportion of triple-negative breast cancers. The findings suggested that South Indian women in the South Canara district, more often have genetically driven, histologically aggressive breast carcinoma at young age, likely to be less susceptible to conventional hormonal and targeted antibody treatment.

In a study conducted by Desai et al which included 798 breast carcinoma cases in Indian population it was observed that $32.6 \%$ were ER positive and $46.1 \%$ were PR positive ${ }^{15}$. ER, PR immunoreactivity increased with advancing age and correlated with presence of elastosis. Infiltrating lobular, mucinous and mixed tumors were more frequently ER, PR positive. High grade infiltrating duct carcinoma, pure comedo DCIS and medullary carcinomas were predominantly ER, PR negative. The presence of necrosis and lymph vascular invasion showed an inverse relationship with ER and PR reactivity.

As studies showed among 40 patients 21 (52.5\%) had left breast carcinoma which is said to be mostly these women were left handed.

In our study luminal A subtype seems to be more common .As we know luminal A subtype tumors had a long term risk of distant metastatic disease which can be reduced by hormonal treatment. And these treatment modalities are well acceptable for the patients visiting in this charitable hospital. Second most common subtype was found Triple negative breast cancer (TNBC) which is more notorious than any other verities as this category has limited schedules for treatment after recurrence and has poor survival. Survival study of triple negative and non triple negative breast cancer in a Brazilian cohort showed 5 years overall and disease free survival is $62.1 \%$ and $57.5 \%$ in TNBC patients where it is $80.8 \%$ in non TNBC patients ${ }^{15}$. 


\section{CONCLUSION}

But for Lack of proper screening most of the patients came to us at stage IV. We need a proper screening at hospital and slum areas so that patients can be diagnosed at earlier stages. Moreover if we can collect more data of patients we can better describe the pattern of molecular subtypes of breast cancer patients of Chattogram zone. For that we need proper facilities for immunohistochemistry in Chittagong.

\section{DISCLOSURE}

All the authors declared no competing interest.

\section{REFERENCES}

1. Justo N, Wilking N, Jönsson B, Luciani S, Cazap E. A Review of Breast Cancer Care and Outcomes in Latin America. Oncologist. 2013;18(3):248-256.

2. Bhikoo R, Srinivasa S, Tzu-Chieh Yu, Moss D, Hill G A. Systematic Review of Breast Cancer Biology in Developing Countries (Part 1): Africa, the Middle East, Eastern Europe, Mexico, the Caribbean and South America. Cancers (Basel). 2011;3(2):2358-2381.

3. Ghoncheh M, Pournamdar Z, Salehiniya H. Incidence and Mortality and Epidemiology of Breast Cancer in the World. Asian Pac J Cancer Prev. 2016;17:43-46.

4. Global cancer observatory. www.gco.iarc.fr. [Google Scholar]).

5. Molecular Classification of Breast Carcinoma: From Traditional, Old-Fashioned Way to A New Age, and A New Way. J Breast Health. 2015;11(2): 59-66.

6. Edge SB, Byrd DR, Compton CC, Fritz AG, Greene FL, Trotti A, eds. AJCC cancer staging manual. 7th ed. New York, NY: Springer. 2009; 347-376.

7. Goldhirsch A, Winer EP, Coates AS, Gelber RD, Piccart-Gebhart M, Thürlimann B, Senn HJ. Panel members- Personalizing the treatment of women with early breast cancer: Highlights of the St Gallen International Expert Consensus on the Primary Therapy of Early Breast Cancer. Annals of Oncology. 2013;24:2206-2208.

8. National Cancer Registry Programme, Indian Council of Medical Research. Leading sites of cancer. In, Consolidated Report of Population Based Cancer Registries 2001-2004, Incidence and Distribution of Cancer. Bangalore: Coordinating Unit, National Cancer Registry Programme (ICMR). 2006;8-30.

9. Onitilio AA, Engel JM, Greenlee RT, Mukesh BN. Breast Cancer Subtypes Based on ER/PR and HER2 expression: Comparison of Clinicopathologic Features and Survival. Clin Med Res. 2009;7(1-2):4-13.

10. Rosai J, editor. Breast. In: Rosai and Ackerman’s Surgical Pathology, $9^{\text {th }}$ ed. St. Louis: Mosby. 2004;1764-1876.

11. Ruibal A, Arias JI. Histologocal grade in breast cancer: Association with clinical and biological features in a series of 229 patients. Int J Biol Markers. 2001;16:56-61.

12. Onitilo AA, Engel JM, Greenlee RT, Mukesh BN. Breast cancer subtypes based on ER/PR and Her2 expression: Comparison of clinicopathological features and survival. Clinical Medicine And Rapid Release 2009 February. Available from: URL: http://www.clinmedres. org/cgi/ content/ abstract/cmr. 2008;1:825.

13. Thike AA, Chng MJ. Immunohistochemical expression of hormone receptors in invasive breast carcinoma: Correlation of results of $\mathrm{H}$ - score with pathological parameters. Pathology. 2001;33:21-25. 